\title{
Datación e identificación de libros impresos, manuscritos y obras de arte a través de las filigranas papeleras
}

\author{
Ma Dolores Díaz De Miranda y MaCías, O.S.B.
}

(Monasterio de Sant Pere de les Puel·les. Taller de Restauración de Documento Gráfico. Barcelona)

\section{Gerard VAN THIENEN}

(Koninklijke Bibliotheek de La Haya, Países Bajos. Holanda)

\section{Resumen}

Este artículo muestra cómo la identificación del papel a través de las filigranas puede permitir datar con precisión, o acotar en un corto espacio de tiempo, el año de producción de un libro, de un manuscrito o de una obra de arte; e, incluso, puede llegar a determinar el lugar de la edición o el impresor

Para el cotejo y estudio de unos y otros papeles es imprescindible la creación de bases de datos que recojan de forma sistematizada las características de los papeles y sus filigranas. En este sentido se nuestra la eficacia de la base de datos WIES (sobre el papel de los incunables españoles) y PFES (sobre cualquier clase de papel de los archivos y bibliotecas españolas). Sin embargo, este tipo de estudios, tiene límites provenientes de la misma producción del papel, de la reproducción de la filigrana o de los datos que otros autores dan de ella, como se muestra a través de los ejemplos seleccionados.

Palabras clave: Datación papel; Filigranas papeleras. 


\section{Dating and identification of printed books, manuscripts and works of art by paper evidence and watermarks}

Abstract

This article shows that identification of paper by watermarks makes it possible to date or ascribe a narrower date to the production year(s) of a printed book, ms, woa. For printed books it even may be possible to determine place of printing and printer.

For the collation and study of all kinds of paper, the creation of databases, with systematic description of the characteristics of paper and watermarks is essential. The effectiveness of database WIES (the watermarks in incunabula printed in Spain) and PFES (on all paper files and libraries Spanish) is demonstrated here. Of course these studies are limited, because of the identification of different paper sorts, the way of reproducing the watermarks (tracing, rubbing, photo), and the data from different authors, as demonstrated in the selected examples.

Keywords: Dating paper; Watermarks.

La identificación del papel a través de las filigranas con la finalidad de datar y ubicar el lugar de producción de un libro impreso, un manuscrito o una obra de arte es un procedimiento del que se tienen conocimiento desde finales del siglo dieciocho. Sin embargo, la experiencia muestra que frecuentemente con papeles anteriores al siglo XVIII pueden darse equívocos a causa de la imprecisión y la deficiente rigurosidad en la recogida de los datos.

En el presente artículo se ofrece una metodología de análisis del papel y de la filigrana que logra evitar o corregir dichos equívocos. La aportación más importante y novedosa, de esta propuesta metodológica, es que el análisis de la hoja de papel se plantea desde el pliego salido de la forma y no sólo desde la filigrana, tal como se ha venido haciendo desde el descubrimiento de las filigranas hasta la actualidad. ${ }^{1}$ La base de datos PFES es el fruto de esta formulación metodológica. Para el caso de los libros impresos cuyas ediciones y números de ejemplares conservados son limitados puede ser suficiente el análisis de la filigrana. Para estos casos la base de datos WIES -de las filigranas de incunables españoles-- es sin duda alguna la que logra el objetivo de poder datar ediciones de incunables españoles sin ningún tipo de referencia a través del cotejo de sus filigranas con las de ejemplares perfectamente identificados.

\footnotetext{
${ }^{1}$ Descubrimiento que se atribuye al polaco Johann Samuel Hering en 1736.
} 
Con todo se habrá de tener en cuenta que este tipo de estudios tiene límites provenientes de la misma producción del papel, de la reproducción de la filigrana o de los datos que otros autores dan de ella. A través de los cuatro ejemplos que se ofrecen se muestra la metodología que se propone, se constata los límites del cotejo con otras filigranas de las cuales no se tienen los datos que serían deseables y se demuestra la validez de las bases de datos WIES y PFES para este tipo de estudio.

\section{La base de datos Watermarks in incunabula printed in España, WIES}

La página Watermarks in Incunabula printed in España -WIES-, hecha por Gerard van Thienen, ${ }^{2}$ es un sitio web provisional que, desde noviembre del 2008, presenta 6.000 frotados de filigranas de los incunables impresos en España de un total, aproximadamente, de 9.000 frotados que el autor ha recogido tras visitar más de cien bibliotecas, especialmente, españolas y de otros países como los Estados Unidos, Inglaterra, Múnich, París, San Petersburgo, Viena, Portugal, Bélgica, Holanda, etc. Está previsto ir introduciendo en ella la totalidad de los frotados.

Su finalidad es atribuir fechas más precisas a los incunables españoles que están sin datar, aproximadamente unas 500 ediciones, por medio de la similitud de sus filigranas con las de los incunables que están datados, también unas 500 ediciones. A su vez sirve para hacer estudios comparativos entre la similitud de los papeles datados de los incunables españoles con los papeles de los incunables sin datar impresos en otros países, tal es el caso de algunos incunables ingleses que utilizan los mismos tipos de papel genovés que los españoles.

En este sitio web, ${ }^{3}$ las imágenes están organizadas por el número de catálogo bibliográfico, en su mayoría por el IBE o HBI y algunos otros, como BMC X y Goff. ${ }^{4} \mathrm{Al}$ pulsar sobre estos números se accede a las imágenes de las

\footnotetext{
2 http://www.bernstein.oeaw.ac.at/databases/wies/index.html.

3 También se puede acceder a WIES desde la KB (Koninklijke Bibliotheek, National Library of the Netherlands), http://watermark.kb.nl/default/search/advanced/; logrando más posibilidades de búsqueda: motivo, ciudad de impresión, impresor...

4 (IBE) Francisco GARCÍA CRAVIOTTO (coord. y dir.), Catálogo General de Incunables en Bibliotecas Españolas, Madrid, Biblioteca Nacional, 1989-1990. 2 v.; IBE (Adiciones I), Julián MARTÍN ABAD, Catálogo General de Incunables en Bibliotecas Españolas CIE (Adiciones y correcciones. I, Madrid, Biblioteca Nacional, 1991; IBE (Adiciones II). Julián MARTíN ABAD, Catálogo General de Incunables en Bibliotecas Españolas (IBE). Adiciones y correcciones. II, Madrid, Biblioteca Nacional, 1994; (HBI) Konrad HAEBLER, Bibliografía Ibérica del siglo XV. Enumeración de todos los libros impresos en España y Portugal hasta el año de 1500, con notas críticas, La Haya y Leipzig, 1903-1917, 2 v.; (BMC X) London. Catalogue of books printed in the XVth century now in the British Museum: Part X: Spain. Portugal, London, Published by the Trustees of the British Museum, 1971; (Goff) Goff, FREDERICK RICHMOND, Incunabula in American
} 
filigranas reproducidas de esa edición, la imagen se ofrece en dos tipos de carpeta. La primera carpeta, "Folder 1", contiene la imagen de la filigrana y en la parte inferior indica el número de corondeles a la izquierda y a la derecha de la misma, en la superior figuran las siglas de la biblioteca a la que pertenece el ejemplar, su ubicación en el volumen y su referencia con el catálogo. En la segunda, "Folder 2" aparece la referencia bibliográfica numérica y dos números más que hacen relación al número de frotado de ese ejemplar dado por el autor. A los pies de la imagen, una regla milimetrada nos permite deducir las dimensiones reales de la filigrana. Los datos sobre el incunable como el autor, el título y el pie de imprenta, se pueden consultar en los libros de referencia impresos (HBI, IBE, etc.). También, se puede obtener directamente esta información por medio de un vínculo, que tiene la página web con la base de incunables ISTC de la Biblioteca Británica. ${ }^{5}$

Consta de un apéndice con la representación de 353 tipologías de filigranas. ${ }^{6}$ A cada filigrana se le da una letra (la correspondiente a la clasificación del IPH) y una cifra, la sucesión de éstas presentan intervalos que el autor ha dejado sin cubrir para futuras adiciones. Sólo el grupo de la mano tiene dos sub-clasificaciones: mano sin figura y mano con figura. En una tabla se encuentra la correlación de los números dados a las filigranas con el correspondiente al catálogo de la British Library.

\section{La base de datos papel y filigranas en España, PFES}

PFES (Papel y Filigranas en España) es una base de datos que recoge las características de los papeles y sus filigranas utilizados en la documentación española a lo largo de la historia, y es el fruto de la base de datos El papel y la filigrana en la documentación española creada en la tesis doctoral de $\mathrm{M}^{\mathrm{a}}$ Dolores Díaz de Miranda, defendida en la Universidad de Barcelona en enero de 2013.7

En junio de 2013 PFES entró a formar parte del Portal Bernstein The Memory of Paper. ${ }^{8}$ Su inclusión ha supuesto tener que reducir el número de campos de la originaria base de datos a las posibilidades de dicho Portal, actualmente se está trabajando para ir introduciendo los campos omitidos. Por

libraries. A third census of fifteenth-century books recorded in North American collections. Reproduced from annotated copy maintained by... New York, 1973

${ }^{5} \mathrm{http}: / /$ www.bl.uk/catalogues/istc.

${ }^{6} \mathrm{G}$. van THIENEN, A. ENDERman y Ma D. DíAz DE Miranda, «El papel y las filigranas de los incunables impresos en España, a través de los diversos ejemplares conservados en las bibliotecas del mundo», Syntagma, Revista del Instituto de Historia del Libro y de la Lectura, 2, (2008) pp. 239-61.

${ }^{7}$ Con el título: Análisis y desarrollo de una base de datos para el estudio del papel y de las filigranas: fuente para la elaboración de la historia del papel en España, (inédita).

${ }^{8}$ http://www.memoryofpaper.eu:8080/BernsteinPortal/appl_start.disp. 
este motivo se comentará los campos de Elpapel y la filigrana en la documentación española.

La aportación más importante de esta base de datos es que no limita el estudio del papel sólo a la filigrana, si no que abarca una visión global del pliego salido de la forma. Así se logra reconstruir las formas papeleras y proporcionar información sobre el molino productor, las vías de comercialización y los usos del papel, entre otros. Este planteamiento es la base de la tesis doctoral citada. Con esta base de datos se ha resuelto una carencia común de todos los estudios sobre filigranas realizados hasta la actualidad.

Los 117 campos que componen esta base de datos se estructuran en función de: -la filigrana; -la hoja de papel; -la forma; -los datos de identificación; -el molino papelero y fabricante; -las vías comerciales; -el papel árabe y -la bibliografía. Están distribuidos en diez formularios que forman las pestañas de la base de datos, las cuales siempre están acompañadas de la imagen de la filigrana y el esquema de su ubicación en el papel.

\section{Ejemplos de identificación y datación}

\section{Codex 184 a y b del Archivo Capitular de la Catedral de Barcelona}

Este codex contiene el canto de la Sibila en latín y en catalán. Esta última lengua es la versión musicalizada más antigua que se conserva. El canto de la Sibila en catalán se encuentra en el archivo de la catedral de Barcelona en dos originales manuscritos del siglo XV y en uno impreso del siglo XVI. ${ }^{9}$ La versión catalana del canto de la Sibila del códex 184 no parece que provenga directamente de la versión latina de este mismo códex, sino de una versión en lengua occitana. Además, en esta versión catalana el canto se canta en los maitines del Sábado Santo en vez de los maitines de Navidad, caso probablemente único en el mundo.

Los cuarenta y dos cuadernos que forman este códex originalmente no constituían una unidad solidaria, lo que facilitó su dispersión entre la documentación del archivo. A partir de las últimas décadas del siglo pasado se fueron reuniendo, según se iban hallando, en dos cajas identificadas como: «Fragmenta codicum: 7 (a) y 7 (b). Libri liturgici. Leccionarium s. XV».10

\footnotetext{
9 De los originales manuscritos uno es del que ocupa este estudio y el otro son las Constitutiones synodales ecclesiae barchinonensis, del año1415, en el folio 35 está el texto del canto de la Sibila. En la versión impresa el canto de la Sibila, está en los folios $285 \mathrm{v}-286$ del Ordinarium Barcinonense del año 1569.

${ }^{10}$ La mayor cantidad de cuadernos fue encontrada entre 1972 y 1974 por Miquel dels Sants Gros y Edouard Jeauneau.
} 
El estudio del papel dio como resultado trece filigranas diferentes. Se buscaron las correspondencias de estas filigranas con otras datadas, utilizando como correspondencia válida para la datación del papel aquellas filigranas cuyas semejanzas permitían deducir que el papel salió del mismo molino o de la misma área papelera que las de este códex. ${ }^{11}$ De las trece filigranas sólo siete valieron para el fin perseguido. Entre ellas se han encontrado:

- Una filigrana idéntica (fil. $1478.11 \mathrm{del} \mathrm{ACV)} \mathrm{a} \mathrm{la} \mathrm{filigrana} \mathrm{del} \mathrm{carro} \mathrm{con}$ ruedas 000768 (PFES), perteneciente a un documento del año 1425 del Archivo de la Catedral de Valencia.

- Otra filigrana idéntica a la 000769 (PFES) -monte de tres picos dentro de un círculo surmontada por una cruz-, en el Archivo del Monasterio de Sant Pere de les Puel-les de Barcelona (AMSPP) en el Llibre de Abadesses del año 1426 (filigrana 000780, PFES) y otra filigrana similar en un Llibre Notarial del año 1425 del Archivo del Monestir de Sant Joan de les Abadesses de Gerona (AMSJA), filigrana 000792 (PFES). El dibujo de esta filigrana (000792) es prácticamente idéntico al de la filigrana 000769,12 sin embargo la distancia entre los corondeles de estos papeles no es la misma y la filigrana se coloca a mano derecha de la forma, mientras que la filigrana 000769 lo hace a la izquierda; podrían ser filigranas gemelas, sean o no lo sean, se ha de concluir que no son filigranas salidas de la misma forma papelera, pero sí del mismo molino.

-Una filigrana similar a la que representa una corona, 000774 (PFES), en un documento del año 1424 de Lucca dos (fil. 4.714 de Briquet) y otras en el Llibre de Abadesses del año 1426 (fil. 000781 y 000782).

- De otro libro de Abadesses del año 1426-1427 una filigrana similar a la 000773 (PFES), que representa una rueda de molino.

- Una filigrana idéntica (fil. 000793) a la filigrana de montaña de tres picos, 000777 (PFES) en el Llibre Notarial del año 1425 del archivo del Monestir de Sant Joan de les Abadesses de Gerona, y dos filigrana similar en el Llibre Notarial del año 1424 del mismo archivo, filigrana 000790 y 000794 (PFES).

Todas las correspondencias que se han encontrado se sitúan en documentos fechados entre 1415 y 1429. El papel se cree que proviene de Italia, algunos de ellos probablemente de la Toscana y se distribuye ampliamente en España, al menos por Valencia, Barcelona y Girona. Como

\footnotetext{
11 La relación se establece en orden a que sean filigranas salidas de la misma forma (filigranas idénticas), que sean filigranas salidas del par de formas utilizadas simultáneamente por el maestro de tina (filigranas gemelas), que sean filigranas hechas en el mismo molino papelero (filigranas similares), aquellas que son muy probablemente del mismo molino sin poderlo asegurar con total certeza (filigranas convergentes) y que probablemente no se hicieran en el mismo pero quizá sean de la misma área papelera (filigranas paralelas).

${ }^{12}$ Las pequeñas variaciones entre ambas se pueden atribuir al proceso de la elaboración manual de cada pliego de papel.
} 
conclusión, se puede decir que ante la existencia de filigranas idénticas y similares, este códex ha sido elaborado entre 1424-1426. Debiendo retrasarse un cuarto de siglo la datación del códex, ya que hasta el momento era considerado de mediados del siglo $\mathrm{XV}^{13}$. Más abajo se detallan estás correspondencias.

Yunque. Filigrana 000766 (PFES)

Filigrana convergente: - 1423, Udine (Italia). PICCARD, No.122.61614

Filigranas paralelas: - 1425, Danzig (Alemania). PICCARD, $\mathrm{N}^{\circ} .122 .603$

- 1427, Marienburg (Alemania). PICCARD, $\mathrm{N}^{\circ} .122 .621$

Rueda. Filigrana 000773 (PFES)

Filigrana similar: - 1426-1427, Barcelona. Filigrana 000779 (PFES) ${ }^{15}$

Filigrana convergente: - 1429, Lorena, Francia. PICCARD, nº 122.928

Montaña de tres picos con una cruz encima. Filigrana 0000777 (PFES)

Filigrana idéntica: $\quad$ - 1425, Gerona. Filigrana 000793 (PFES) ${ }^{16}$

Filigrana similar: - 1424, Gerona. Filigrana 000790 y 000794 (PFES) ${ }^{17}$

Filigrana convergente: - [1424-1426], Barcelona. Filigrana 000778 $(\mathrm{PFES})^{18}$

Filigrana paralela: $\quad-1423$, Florencia (Italia). BRIQUET, $\mathrm{n}^{\circ} 11.725$.

\footnotetext{
13 Josep BAUCELLS i REIG, «El cant de la Sibil-la a la catedral de Barcelona. (Edició dels textos i estudi de la segona época de la representació: ss. XV-XVI)», Revista Catalana de Teologia, 6 (1981), p. 183.

${ }^{14}$ Las bases de datos: PICCARD, PFES, WIES, BRIQUET, ACV y LIKHACHEV se pueden consultar en línea: http://www.memoryofpaper.eu:8080/BernsteinPortal/appl_ start.disp.

${ }^{15}$ Llibre de Abadesses $N^{\circ} 73$, fol. 77v-99, Archivo del Monasterio de Sant Pere de les Puel·les (AMSPP).

${ }^{16}$ Llibre Notarial. Notari Joan de Coll. Sant Joan de les Abadesses. Anys 1425, fol. 79v-70. Signatura: G14. Nota decimoquinta. Arxiu del Monestir de Sant Joan de les Abadesses (AMSJA).

17 AMSJA, Llibre Notarial. Notari Joan de Coll. Sant Joan de les Abadesses. Anys 1424. Signatura: G14. Nota decimoquarta.

${ }^{18}$ Filigrana del Codex 184 del Archivo Capitular de la Catedral de Barcelona, fol. 493-494v
} 
Montaña de tres picos dentro de un círculo surmontado por una cruz. Filigrana 0000769 (PFES)

Filigrana idéntica: $\quad$ - 1426, Barcelona. Filigrana 000780 (PFES) ${ }^{19}$

Filigrana similar: $\quad-1425$, Gerona. Filigrana 000792 (PFES) ${ }^{20}$

Filigranas convergentes:- 1418, Gerona. Filigrana 000796 (PFES ${ }^{21}$

- 1425, Barcelona. Reproducida por VALLS22

Cuerno de música. Filigrana 000770 (PFES)

Filigrana gemela: $\quad-$ [1424-1426], Barcelona. Filigrana 000771 PFES) ${ }^{23}$

Filigranas paralelas: $\quad-1418$, Valencia. ACV, $\mathrm{n}^{\circ}$ XV29.

- 1420-1429. LIKHACHEV, no 2.418

- 1425-1426. LIKHACHEV, no 905

Corona. Filigrana 000774 (PFES)

Filigrana similar: $\quad-1424$, Lucca (Toscana, Italia). BRIQUET, $\mathrm{n}^{\circ}$ 4.714

- 1426, Barcelona. Filigrana 000781 y 000782

$(\mathrm{PEFS})^{24}$

Carro con dos ruedas. Filigrana 000768 (PFES)

Filigrana idéntica: - 1425, Valencia. ACV, no 1478.11

Filigranas convergentes:- 1421, Siena. BRIQUET, no 3.543

- 1415, Valencia. ACV, $n^{\circ} 1478.9$

19 AMSPP, Llibre de Abadesses $N^{\circ} 72$, fol.23rbis - [02v].

20 AMSJA, Llibre Notarial. Notari Joan de Coll. Sant Joan de les Abadesses Anys 1425, fol. 69v-80. Signatura: G14. Nota decimoquinta.

${ }^{21}$ AMSJA, Llibre Notarial. Notari Joan de Coll. Sant Joan de les Abadesses Anys 1418, fol. 21v-18. Signatura: G12. Nota octava.

${ }^{22}$ Libro de Cuentas y Testamentos (1425-1434). Notaria de Antoni Brocard. Archivo Histórico de Protocolos de Barcelona. Historia del papel en España. Vol. II. Madrid, Empresa Nacional de Celulosa, 1980, filigrana, 205, pp. 160 y 230.

${ }^{23}$ Filigrana del Codex 184 del Arcbivo Capitular de la Catedral de Barcelona, fol. 238-249v.

24 AMSPP, Llibre de Abadesses $N^{o} 72$, fol. 34v-41 (filigrana 000781, PFES) y fol. 45-30v (filigrana 000782, PFES.

Titivillus, ISSN 2387-0915, ISSN-e 2603-9966, 1 (2015), pp. 101-119 


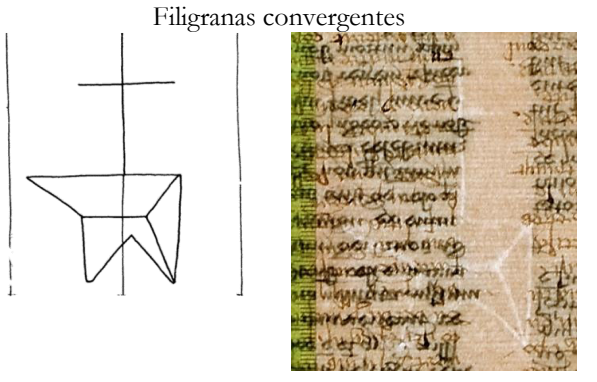

1423, Udine (Italia).PICCARD, 122.616
Barcelona. 000766 (PFES)

Filigranas idénticas

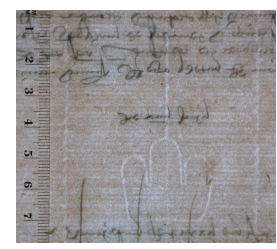

Gerona, 1425. 000793 (PFES)

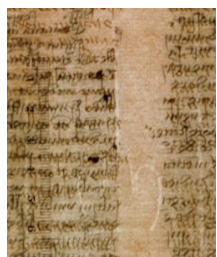

Barcelona. 000777 (PFES)

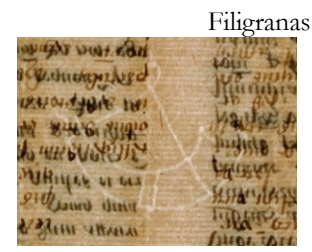

Barcelona. 000770 (PFES)

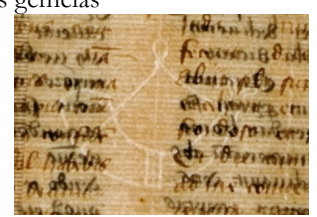

Barcelona. 000771 (PFES)

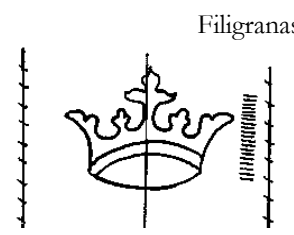

1424, Lucca (Toscana, Italia). BRIQUET,4.714

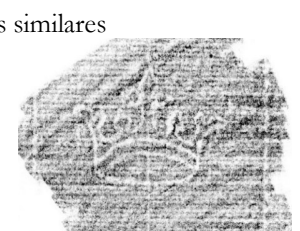

Barcelona. 000774 (PFES)
Filigranas similares
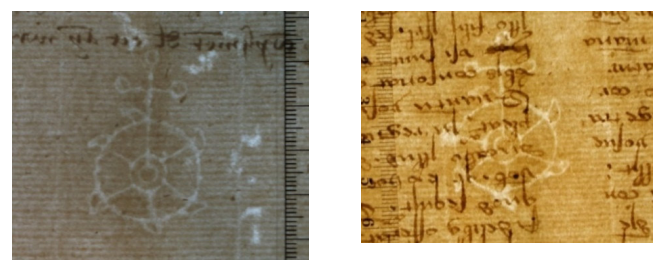

1426, Barcelona. 000779 (PFES)

Barcelona. 000773 (PFES)

Filigranas idénticas
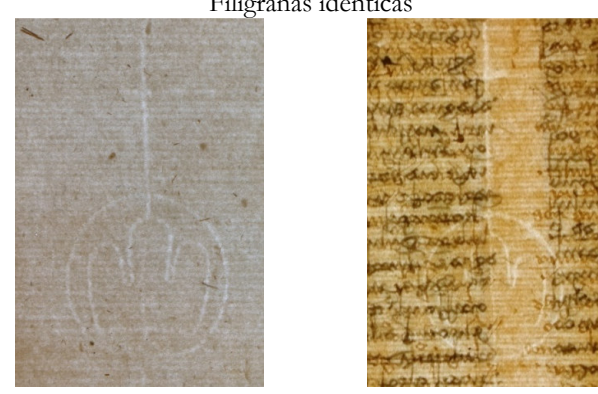

1426, Barcelona. 000780 (PFES)

Barcelona, 000769 (PFES)

Filigranas idénticas
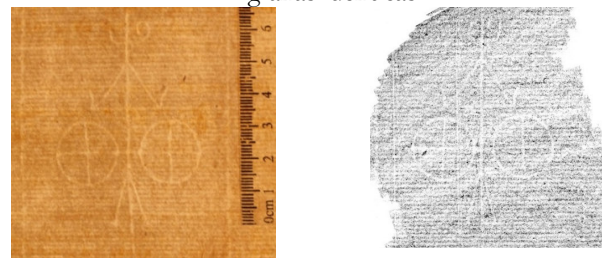

1425, Valencia. ACV, $\mathrm{n}^{\circ}$

Barcelona, 000768 (PFES) 1478.11

Filigranas similares

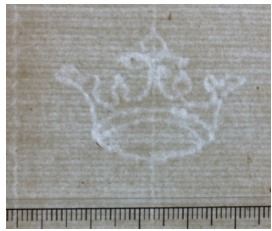

1426, Barcelona. 000781 (PFES)

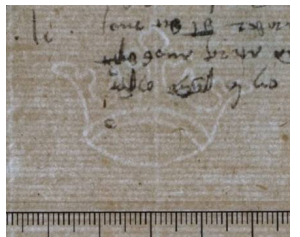

1426, Barcelona. 0007812 (PFES) 


\section{Libro de las Frases o Sharb al-Yumal de Ibn Al-Fajjar al-Bayri}

Este manuscrito pertenece a la colección de Manuscritos árabes del archivo de Sacromonte de Granada, es una summa gramatical andalusí, en la que se recoge la obra titulada Sharh al-Yumal, del gramático almeriense AlFajjar al-Bayri, afincado en la capital granadina durante el siglo XIV. El copista, que no contó con el ejemplar completo, añadió para compensar la ausencia del final de dicha obra un opúsculo de no más de 15 folios, a modo de rápida guía de la ciencia de la sintaxis árabe ${ }^{25}$ La obra termina con el colofón sin data alguna.

El cuerpo del libro está formado por 245 hojas de papel verjurado, de 293 $\mathrm{mm}$ de alto, $210 \mathrm{~mm}$ de ancho y 180-350 micras de espesor. Tiene tres tipos de filigranas, la primera representa una iglesia y las otras dos son una mano con cuatro dedos juntos y el pulgar separado, encima tienen una estrella de cinco puntas. Para la datación del papel se ha utilizado la filigrana de la iglesia, ${ }^{26}$ ya que las filigranas de la mano son filigranas muy abundantes en el siglo XV y principios del $\mathrm{XVI}^{27}$ y la variedad de tipos es tan grande que en muchos casos las diferencias son poco claras. Las dos filigranas de la mano de este libro encajan en los tipos de filigranas de la mano de finales del siglo XV.

La filigrana de la iglesia se encuentra del bifolio $1 / 8$ al folio $70 \mathrm{y}$ del bifolio 67/176 al 177/186 (decimoctavo y decimonoveno cuadernillo). Su espesor oscila entre 220 - 250 micras, hay entre 10 y 11 corondeles por bifolio y entre 20 y 22 puntizones en un espacio de $20 \mathrm{~mm}$.

Se han encontrado este tipo de filigranas en diversos documentos, la variante más importante entre ellas es la sustitución de la cruz del campanario por una tau, aunque se considera que todas han podido salir de un mismo molino o área papelera. A continuación se relacionan estos documentos:

\footnotetext{
${ }^{25}$ Cfr. Juan Pablo Arias TOrRes, «Una suma de los estudios gramaticales en el al-Āndalus hacia finales del siglo XV», en David TORRES IBÁÑEZ y Teresa ESPEJO ARIAS (coord.). Comentario al Libro de las Frases (Sharh al-Yumal) de Ibn Al-Fajjar Al-Bayri. Sevilla, Consejería de Cultura, 2010, ed. digital, pp. 306-11.

${ }^{26}$ Los datos sobre esta filigrana nos fueron solicitados por Teresa Espejo, profesora de la Universidad de Granada y responsable de la restauración realizada a este libro. Los trabajos de investigación y de restauración fueron publicados en Comentario al Libro de las Frases (Sharb al-Yumal) de Ibn Al-Faijar Al-Bayri. Sobre la datación de la filigrana en el artículo «Estudio codicológico del manuscrito XVI de la Abadía de Sacromonte: Aportaciones para una arqueología libraría», se produjo un error tipográfico señalado por la autora en la fe de erratas: "en la página 320, donde dice años 1740 y 1786, debe decir años 1470 y 1486".

${ }^{27} \mathrm{M}^{\mathrm{a}}$ D. DíAz De MiRAnda y G. van THIENEN, «Watermarks in Spanish Manuscripts and Incunabula in Piccard-Online ». En: Wasserzeichen und Filigranologie. Beiträge einer Tagung zum 100. Geburtstag vo Gerhard Piccard (1909-1989), Stuttgart, Landesarchiv Baden-Württemberg, Hauptstaatsarchiv Stuttgar, 2011, pp. 101-21.
}

Titivillus, ISSN 2387-0915, ISSN-e 2603-9966, 1 (2015), pp. 101-119 
- 1470, Oviedo, Principado de Asturias. Filigranas 000380 y 000381 (PFES). Se trata de dos filigranas gemelas encontradas en un Protocolo Notarial -21.11.1470/07.09.1471- del Fondo de Valdecarzana del Archivo del Real Instituto de Estudios Asturianos -RIDEA-. Corresponderían al par de formas utilizadas por el maestro de tina en la elaboración del papel.

- 1470, Villareal, Castellón de la Plana. Filigrana no 105 del Archivo Municipal de Villareal, reproducida por José $\mathrm{M}^{\mathrm{a}}$ Doñate Sebastia. ${ }^{28}$

- 1482, 12 de noviembre, Zaragoza. WIES, IBE 5668.47 a $50 .{ }^{29}$ Expositio super toto psalterio, Juan de Torquemada. Impresor: Paul Hurus y Johann Planck.

- 1483, Villareal, Castellón de la Plana. Filigrana no 105 del Archivo Municipal de Villareal, reproducida por José $\mathrm{M}^{\mathrm{a}}$ Doñate Sebastia. ${ }^{30}$ Esta filigrana tiene sobre el campanario una tau.

- 1484, 11 de noviembre, Huete, Cuenca. WIES, IBE 2068.07, 08 y 11. Ordenanzas reales, Alonso Díaz de Montalvo. Impresor: Alvaro de Castro. Tiene sobre el campanario una tau.

-1485, 12 de marzo, Burgos. WIES, IBE 2820.04. Grammatica latina, Andreas Guterrius. Impresor: Fabrique de Basilea, ejemplar de la Biblioteca Nacional, Madrid. Tiene sobre el campanario una tau.

-- 1485, 23 de agosto, Huete, Cuenca. WIES, IBE 2070.17 y 18. Ordenanzas reales. Alonso Díaz de Montalvo. Impresor: Alvaro de Castro. Tiene sobre el campanario una tau.

- [1485], Burgos. WIES, IBE 1764.01. Coplas de mingo revulgo glosado por Fernando de Pulgar. Fernando de Pulgar. Impresor: Fabrique de Basilea. Tiene sobre el campanario una tau.

- [1485], Salamanca. WIES, IBE 4307.01 y 02. Compendium grammaticae, sive Thesaurus pauperum, sive Speculum puerorum, Juan de Pastrana. Impresor: Nebrissensis, 'Introductiones'. Tiene sobre el campanario una tau.

- 1486, Córdoba. Filigrana n ${ }^{\circ} 77$ del archivo Municipal de Córdoba (Sección 1, serie 11) reproducida por Ricardo Córdoba de la Llave y Manuel Cerezo Villegas. ${ }^{31}$ Tiene sobre el campanario una tau.

Como conclusión, esta filigrana se encuentra entre los años 1470 y 1486 en documentos distribuidos por toda la geografía española. Puede pertenecer a un único molino papelero, en diversos casos son filigranas gemelas, y el hecho que a partir de 1483 lleven sobre el campanario una tau y no una cruz podría indicar el cambio de formas, desgastadas por el uso. La filigrana estudiada tiene una cruz sobre el campanario lo que permite acotar la elaboración de este papel entre 1470 y 1482. La manufactura del papel es la italiana,

\footnotetext{
28 «Filigranas del archivo Municipal de Villareal», Ligarzas 5 (1973).

29 Todas la filigranas de WIES están reproducidas por Gerard van Thienen, y se encuentran disponibles en línea (http://www.ksbm.oeaw.ac.at/wies/).

30 «Filigranas del archivo Municipal de Villareal», Ligarzas 5 (1973).

31 «Filigranas del Archivo Municipal de Córdoba (1450-1550)». Anales de la Universidad de Alicante. Historia Medieval 6, (1987), p. 422.
} 
manufactura que paulatinamente fue sustituyendo la manufactura hispanoárabe, propia de España; el hecho que no se haya encontrado esta filigrana en papeles de otros países deja abierta la posibilidad de que sea un papel fabricado en España con la técnica italiana.

Libro de las Frases o Sharh al-Yumal de Ibn Al-Fajjar al-Bayri

Estas filigranas podrían pertenecer a un mismo molino papelero

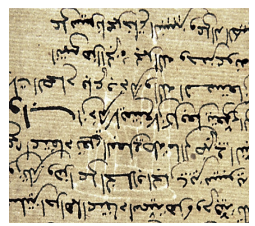

Granada, Libro de las Frases ${ }^{32}$

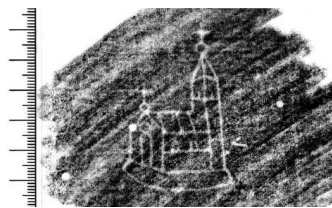

1470, Oviedo. 000382 PFES

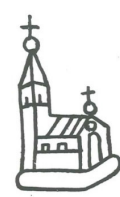

1470, Villarreal (105. Doñate)

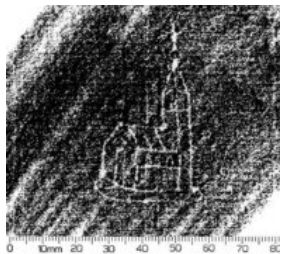

1482, Zaragoza. WIES (IBE 5668.49)

Identificación de material cartográfico de ingenieros militares del siglo XVII

En este ejemplo se propone el caso de la búsqueda e identificación del material cartográfico producido por un grupo de siete ingenieros militares italianos del XVII, ${ }^{33}$ que sirvieron en el ejército de la Monarquía Hispánica en la frontera hispano-portuguesa durante la Guerra de Restauración portuguesa (1640-1668), habiéndose podido documentar entre 1663-1669 en este espacio geográfico, fundamentalmente en Badajoz; a partir de esta última fecha se dispersan, alguno de ellos se desplaza a la frontera catalana, otros a Madrid, las Indias... Este material cartográfico manuscrito sobre papel está muy disperso por instituciones de toda España y el resto de Europa (Suecia, Alemania, Francia, Viena, Italia). Casi todo sin firmar o con firmas inidentificadas. Para su identificación se ha recurrido a fuentes secundarias como las filigranas. Por este motivo, se recibió la reproducción fotográfica de treinta y una filigranas, con la petición de si sería posibles determinar el su origen y datación del papel. ${ }^{34}$

\footnotetext{
${ }^{32}$ Filigrana reproducida por fotografía digital. Esta imagen pertenece a: Comentario al Libro de las Frases..., p. 320.

33 Ventura de Tarragona, Marco Alessandro del Borro, Jerónimo Rinaldi, Lorenzo Possi, Juan Bautista Ruggero, Ambrosio Borsano y Esteban Matteini.

${ }^{34} \mathrm{La}$ información que se ofrece sobre este grupo de ingenieros italianos pertenece a Carlos Sánchez Rubio, "de 4 Gatos". Acaba de ver la luz con el título El Atlas Medici de Lorenzo Possi. 1687 "piante d'Estremadura e di Catalogna», obra escrita por este autor en colaboración con Rocío Sánchez Rubio e Isabel Testón Núñez. La aportación realizada al estudio de Carlos Sánchez se ha basado en los hallazgos y dificultades originados en la búsqueda y
} 
En relación a estas filigranas el descubrimiento más relevante es el caso del Atlas de fortificaciones del Reino de Portugal y Extremadura conservado en el Centro Geográfico del Ejército, con la signatura Ar.G bis-T.6-C.2-50, donde se incluyen trece plantas manuscritas de diversas localidades de la frontera hispano portuguesa. El propio Centro Geográfico las tiene catalogadas como copias realizadas en el siglo XIX de originales anteriores. Se desconoce cuál fue el motivo para esa datación. Incluso, hay varias publicaciones académicas donde se relacionan estudios del atlas otorgándole el siglo XIX como fecha de realización..$^{35}$

El examen del papel y su cotejo con otros papeles sitúa el uso en España de las filigranas entre 1656-1678. Además, por las filigranas de los planos de este atlas se ha podido constatar que el papel proviene del mismo molino papelero que el de otros dos planos manuscritos conservados en el Archivo Militar de Estocolmo. Concretamente las filigranas de siete planos de este atlas $^{36}$ son idénticas a las de dos planos firmados y datados por el capitán Ambrosio Borsano en torno al año 1665 del archivo de Estocolmo. Este dato, junto a los desprendidos de la actividad cartográfica de estos ingenieros militares, permite poder afirmar que el atlas de Madrid no es una copia del siglo XIX sino un original realizado hacia el año 1665.

Las dificultades encontradas en el análisis y cotejo de estas filigranas con otras publicadas por otros autores son una muestra que ejemplifica la problemática más frecuente que entraña este tipo de estudio. Así, a pesar de que las reproducciones de las treinta y una filigranas remitidas eran bastante buenas, diversos factores han dificultado la datación e identificación del papel. En primer lugar, se ha de destacar que parte de las reproducciones fotográficas de las filigranas no se hicieron con un trípode fijo sobre una mesa, y si bien se ha procurado poner una regla al lado de la filigrana, al no estar la cámara en paralelo con la hoja de papel la filigrana y la misma regla presentan ligeras deformaciones de la imagen; al superponer esta imagen sobre otra que se considere que tengan una filigrana idéntica no coincidirá exactamente. En segundo lugar, se ha de considerar las variaciones naturales entre las filigranas salidas de una misma forma papelera, porque al ser su elaboración un proceso manual es imposible que se pueda obtener un idéntico producto. Además, hay que tener en cuenta el desgaste que la forma va sufriendo con el uso; tal como

cotejo de las 31 filigranas recibidas con otras filigranas datas, tal como se expone en este artículo.

${ }^{35}$ Cfr. María CRUZ VILLALÓN (coord.), Ciudades y Núcleos fortificados de la Frontera HispanoLusa. El territorio de Extremadura y Alentejo. Historia y patrimonio, Cáceres, Universidad de Extremadura, 2007.

36 Ar.G bis-T.6-C.2-50 (1)-ALCANTARA, Ar.G bis-T.6-C.2-50 (4) OLIVENZA, Ar.G bis-T.6-C.2-50 (5) ELVAS, Ar.G bis-T.6-C.2-50 (6) ESTREMOZ, Ar.G bis-T.6-C.2-50 (7) CAMPO MAIOR, Ar.G bis-T.6-C.2-50 (11) OUGUELA y Ar.G bis-T.6-C.2-50 (12) ALBURQUERQUE. 
se puede ver en las filigranas idénticas que se reproducen de tres círculos (del Instituto Iberoamericano de Berlín de las villas de Vila Viçosa y Alburquerque) que tienen en el segundo círculo las iniciales GAD y en el tercero la inicial S, la S del tercer círculo aparece medio desaparecida en una de las filigranas.
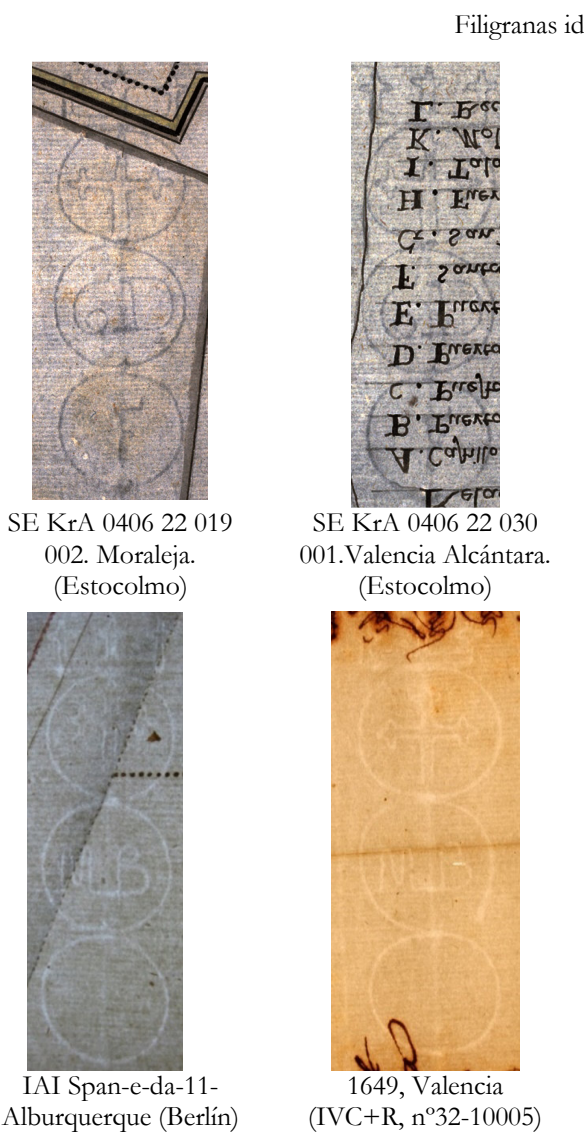

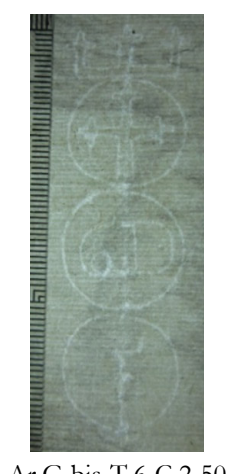

Ar.G bis-T.6-C.2-50

(11) Ouguela

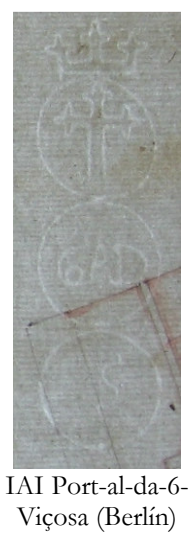

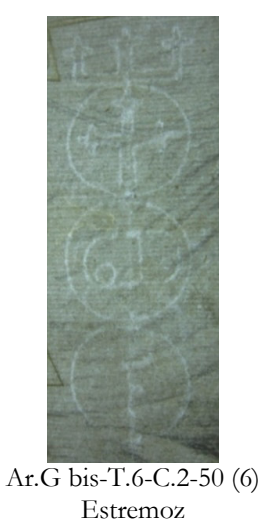

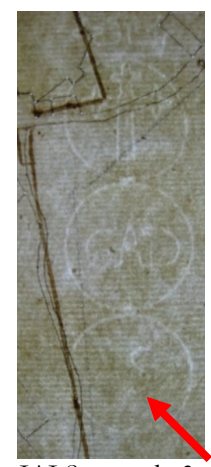

IAI Span-e-da-3-

Alburquerque. (Berlín)

De todas estas consideraciones se concluye que para asegurar que unas filigranas sean idénticas es necesario acompañar su reproducción con otros datos como: las dimensiones de la hoja, el número de corondeles totales, el número de corondeles portadores, la distancia entre ellos, la distancia mínima de la filigrana al corondel derecho e

\footnotetext{
${ }^{37}$ Estas cuatro filigranas han salido del mismo molino papelero y de la misma forma. La reproducción de las ocho filigranas de estos documentos han sido facilitadas por Carlos Sánchez Rubio, “de 4 Gatos”.
}

Titivillus, ISSN 2387-0915, ISSN-e 2603-9966, 1 (2015), pp. 101-119 
izquierdo más próximo y el número de puntizones en $20 \mathrm{~mm}$ o el número de corondeles a la izquierda de la filigrana. ${ }^{38}$

Por último, en el cotejo de una filigrana con otras publicadas hay que considerar las deficiencias de las reproducciones de las filigranas que presentan muchas publicaciones. Principalmente interpretaciones erróneas de la imagen, por ser reproducciones hechas por calco manual, o no estar a escala 1:1. También, los errores de datación realizados por los autores, como el caso comentado anteriormente de la filigrana que reproduce Oriol Valls y que data en 1604. ${ }^{39}$ Esta datación se he de considera improbable en la documentación española, pues tras consultar 250.000 filigranas y encontrar diversos ejemplares de este tipo de filigrana se ha visto que no son anteriores a 1640.

Álbum de grabados de Giovanni Battista Piranesi del Museo de Bellas Artes de Oviedo

Giambattista Piranesi nació en el año 1720 cerca de Venecia. Es considerado uno de los mejores y más prolíficos grabadores de todos los tiempos. Murió el 9 de noviembre de 1778, a los 58 años de edad. Sus hijos Francisco y Laura siguieron grabando, pero a los pocos meses se vieron obligados a emigrar a París por motivos políticos. En esta ciudad continúan la actividad calcográfica, reimprimen la obra de su padre y la enriquecen con nuevos grabados. Entre 1804-1807 se publicó en Paris toda la obra de Giovanni Battista Piranesi con algunos grabados realizados por su hijo Francesco. La obra comprendía 26 tomos encuadernados en 25 volúmenes, el volumen 14 contenía las tres series de grabados que forman el Álbum que estudiamos. Entre 1835-1839 la casa Firmin-Didot adquirió los cobres y los publicó, pero gracias a la intervención del papa Gregorio XVI volvieron a Roma.

\footnotetext{
38 Visión de la filigrana dentro de la hoja de papel que es la principal aportación de la tesis doctoral de $\mathrm{M}^{\mathrm{a}}$ Dolores Díaz de Miranda, como ya se ha comentado.

${ }^{39}$ La filigrana la atribuye a un documento fechado en Toledo en 1604, perteneciente a documentos de la catedral de Toledo. Cfr. VAlLS I SubIRÁ, Oriol, La historia del papel en España. Siglos XVII-XIX, Vol. III, Madrid, Empresa Nacional de Celulosas, 1982, p. 56, filigrana $\mathrm{n}^{\circ} 3$.
}

Titivillus, ISSN 2387-0915, ISSN-e 2603-9966, 1 (2015), pp. 101-119 


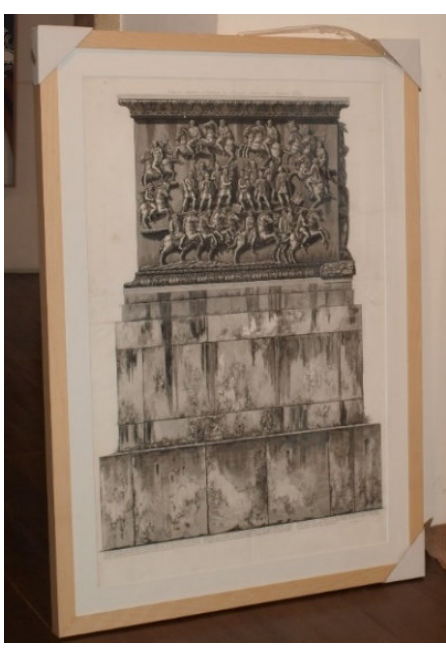

Grabado 23 (25): Stylobates marmoreus consecrationis. A. Pii

El volumen de grabados que se analiza contiene la serie de la Columna Trajana, formada por 21 grabados acabados entre 1773-1774, a la que se añadiría rápidamente la serie de la Columna de Marco Aurelio o Antonina, formada por 2 planchas, y la de la Columna de Antonio Pío, con 5 planchas. La edición conjunta vio la luz sobre el año 1775. ${ }^{40}$ Este Álbum, del que habían sustraído dos grabados, llegó al Museo de Bellas Artes de Asturias del Archivo General de la Administración del Principado de Asturias, al que había llegado a su vez del archivo de la extinguida Diputación. A esta última se supone que fue trasladado en 1936 de la semidestruida Escuela de Bellas Artes de Oviedo. ${ }^{41}$ No se sabe cómo llegó a ella, pero sí que provenía del École Impériale des Beaux Arts/Biblioteque, tal como indica el sello tampón ovalado del último grabado.

Cuando ingresó en el Taller de Restauración del Monasterio de san Pelayo, una de las cuestiones que se debía resolver era determinar a través del papel el año de publicación de estos grabados.

El tipo de papel utilizado es un papel verjurado elaborado a mano y constituido por celulosa natural. Se distinguen dos tipos de papel. Uno de ellos no tiene filigrana y los corondeles se colocan a una distancia de 31-33 $\mathrm{mm}$. El otro, que pertenece a los grabados 23(25) y 24(26), tiene filigrana y los corondeles guardan una distancia de $37-40 \mathrm{~mm}$, en cada $20 \mathrm{~mm}$ hay entre 13 y 14 puntizones. En este papel el pliego, que está cortado, mide unos $550 \mathrm{~mm}$ de alto por 835 de ancho y 350 micras de espesor medio; en el lado derecho del pliego aparece una gran águila bicéfala con una corona (de $159 \mathrm{~mm}$ x 125 $\mathrm{mm}$ ), en el lado izquierdo la filigrana (de $76 \mathrm{~mm}$ x $114 \mathrm{~mm}$ ) designa la calidad del papel, las iniciales del nombre del papelero y su apellido y la provincia o región donde estaba el molino. (Véase la reproducción de la filigrana). ${ }^{42} \mathrm{Se}$

${ }^{40}$ Cfr. El estudio sobre estos grabados de J.A. Calatrava Escobar y la restauración de Ma D. DíAz DE Miranda en: Giovanni Battista Piranesi (1720-1778). Aguafuertes: la Columna Trajana, la Columna de Marco Aurelio (Antonina), la Columna de Antonino Pio, Oviedo, Museo de Bellas Artes de Asturias, 1991, pp. 7-39 y 43-66.

${ }^{41}$ En el lomo de la encuadernación del volumen figuraba dorado: PIRANESI / COLONNE / TRAJANA / ESCUELA / ESCUELA DE BELLAS ARTES / OVIEDO.

${ }^{42}$ En la reproducción de esta filigrana no se representan los corondeles, motivo por el que se quiso examinar la obra original para completar este dato. Se agradece al director del Museo de Bellas Artes de Asturias, el señor Alfonso Palacio Álvarez, la amabilidad y 
trata de un papel de gran formato denominado Petite aigle, las letras FIN, indican que su calidad es papel "fino" y que ha sido fabricado después de 1739. ${ }^{43}$ La inicial T seguida de la inicial M, surmontada por un círculo sobre el cual hay una $\mathrm{T}$ y el apellido DUPUY, inclina a atribuir esta filigrana a ThomasMarie-Joseph Dupuy ${ }^{44}$ (1746-1823), papelero de una familia noble de la localidad de la Grand'Rive y miembro de una de las familias productoras de papel más importante de esta localidad, de la comuna de Ambert, situada en el departamento de Puy-de-Dôme, de la región de Auvergne. ${ }^{45}$
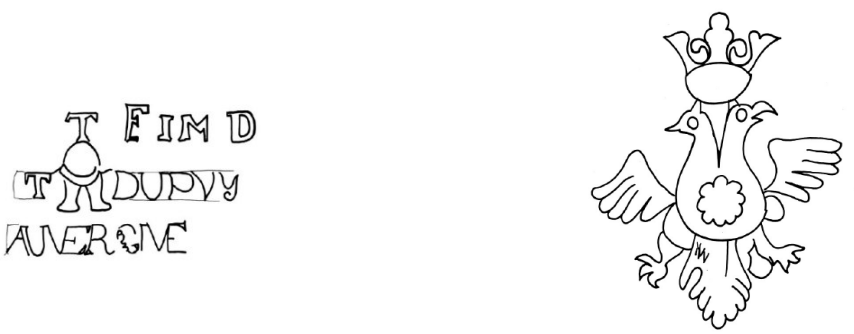

Grabado 23 (25): Stylobates marmoreus consecrationis,.Imp. Antonii Pii. Filg.002266 A y B, PFES

La encuadernación del Álbum era en holandesa con punteras de papel y cubiertas de papel jaspeado color azul verdoso, realizado por el sistema del agua acederas -ácido oxálico- y clara de huevo, las tapas estaban formadas por cartón de celulosa natural. Según Antolín Palomino es un tipo de encuadernación del siglo XVIII - principios del siglo XIX.

En base a estos datos, y considerando que el periodo máximo de tiempo entre la fabricación de un papel y su utilización es de 12 a 15 años como indica

facilidades ofrecidas para poder para examinar las obras. El hecho de que estuvieran enmarcadas y que la obtención de estos datos no fuera imprescindible para la datación del papel, hizo que se fotografiaran las obras enmarcadas, como se puede ver en la imagen que se reproduce de uno de los grabados.

43 Puesto que en este año un decreto real del 27 de enero obligaba a indicar la calidad del papel con todas las letras y no sólo con la inicial, como obligaba el decreto de 1727 para los papeles producidos en Aurvregne. Las tres suerte de papel: F, M y B pasarían a indicarse como: Fin, Moyer y Bulle. Además, en el nuevo decreto de 1739, debía figurar no sólo la inicial del nombre del papelero sino el apellido completo y la provincia donde se ubicaba el molino. Cfr.: Raymond GAUDRIAULT, Filigranes et autres caractéristiques des papiers fabriqués en France aux XVIIe et XVIIIe siècles, Paris, CNRS et J. Telford, 1995, pp. 27 y 32.

${ }^{44}$ Cfr.: Louis APCHER, Une vie de notables auvergnats au cours de trois siecles d'bistoire (1570-à nos jours): les Dupuy de la Grandrive leurs papeteries de la Grandrive et Barot: leur parent, l'Intendant du Canada Claude-Thomas Dupuy, Paris, Saffroy, 1937, p. 100.

${ }^{45}$ Cfr.: Pierre-Claude ReYnard, Histoires de papier: la papeterie awvergnate et ses historiens. Paris, Presses Universitaires Pascal-Blaise, 2001, pp. 140-1 y 150-1. 
Briquet y ratifica Gaudrialt, ${ }^{46}$ se puede concluir que se está ante un ejemplar perteneciente a la publicación realizada por Francesco Piranesi en París entre 1804 y 1807.

\section{Conclusiones}

Para datar y saber el lugar de elaboración de un papel, la filigrana papelera es un elemento que puede aportar información fundamental. La reproducción de la simple filigrana en la mayoría de los casos es claramente insuficiente cuando se trata de papeles anteriores al siglo XVIII. El papel ha de ser estudiado en todo su conjunto, tanto desde sus características físicas como comerciales, económicas, sociales, etc. En un mundo globalizado como el actual y en el que la informática es una herramienta insustituible de comunicación e intercambio de conocimiento, la creación de bases de datos sobre el papel y las filigranas son una herramienta imprescindible para la identificación de papeles de los que no se disponga información documental.

El estudio de las filigranas de los incunables españoles, a través de la base de datos WIES, siguiendo el método de comparación y similitud de las filigranas, permite precisar la fecha de edición de aproximadamente unos 500 ediciones de incunables españoles aún sin datar. El protocolo de análisis del papel y las filigranas que aporta la PFES permite una visión de la hoja de papel que supera la de la simple y sencilla filigrana, siendo capaz de ofrecer los datos necesarios sobre el papel que garanticen un cotejo fidedigno de los papeles y recoger otros datos que explican la distribución y el uso del papel. El análisis de la hoja de papel es imprescindible para los papeles anteriores al siglo XVIII, en los cuales sus filigranas no suelen llevar signos gráficos sobre la identidad del papelero, el lugar de ubicación del molino o el año de fabricación del papel, como se muestra en las filigranas de los cuatro ejemplos expuestos.

En concreto, para el codex 184 de la Catedral de Barcelona la información aportada por la base de datos PFES ha permitido datar la elaboración de este manuscrito, con un alto nivel de precisión entre 1424 y 1426. En el Libro de las Frases o Sharb al-Yumal de Ibn Al-Fajjar al-Bayri se siguió un criterio de selección de los tres tipos de filigranas que tenía su papel, en orden a que los resultaron fueran lo más precisos posibles. Así, se desestimaron las dos filigranas que representaban la mano, por tener un número de variantes muy elevado, y se centró la búsqueda en la filigrana de la iglesia, para cuya datación ha sido fundamental la información aportada por las bases de datos PFES y WIES.

En las filigranas pertenecientes al material cartográfico de ingenieros militares del siglo XVII la existencia en ellas de letras iniciales es indicador de su autoría, pero al día de hoy no se ha descubierto su correspondencia con el

${ }^{46}$ GAUDRIAULT, Filigranes et autres caractéristiques des papiers fabriqués en France, p. 28.

Titivillus, ISSN 2387-0915, ISSN-e 2603-9966, 1 (2015), pp. 101-119 
nombre de los papeleros o el lugar de elaboración. Se debe seguir la misma metodología de análisis de los ejemplos anteriores. Además, su estudio ha hecho evidente que se debe tener en cuenta los límites provenientes de la producción manual del papel, de la reproducción de la filigrana o de los datos que otros autores dan de las filigranas.

La filigrana de principios del siglo XIX de los grabados de Giovanni Battista Piranesi aporta datos más concisos sobre la clase de papel, el nombre del papelero y el lugar de producción, pudiendo realizarse la identificación del papel sin ser tan imprescindible datos precisos sobre la verjura y el pliego salido de la forma.

Por último, se ha de indicar que cuando se estudia el papel de obras impresas de las que se sabe el año, el lugar y el impresor se pueden transpolar estos datos a obras similares con las mismas filigranas; en cambio, para las filigranas de manuscritos no siempre se podrá hacer lo mismo, puesto que estamos ante ejemplares que no son iguales y aunque las filigranas sean idénticas pueden haber sido utilizadas en distintas fechas, como el caso de las filigranas del carro encontradas en la catedral de Barcelona y en la catedral de Valencia. También, en libros impresos, y concretamente en los incunables que se han estudiado a través de WIES y PFES, se ha podido constatar que los ejemplares pertenecientes a la misma edición pueden tener filigranas diferentes entre sí. Como sucede con la filigrana de una serpiente que no se encuentra en el Breviarium secundum congregationis Santi Benedicti de Valladolid (Montserrat, 1500) de la Biblioteca de la Abadía de Montserrat y sí en el de la Biblioteca Pública de Girona. ${ }^{47}$

47 Cfr. Ma D. DíAz DE Miranda, «La imprenta y el papel a través de los incunables montserratinos», IPH Congress Book. 16 (2006), pp 143-57. 